\title{
Macro e micronutrientes na orientação nutricional
}

\section{H世}

para obesidade

Macro and micronutrients in nutritional guidance for obesity

\author{
(D) Fernanda Cristina Carvalho \\ Mattos Magno ${ }^{1}$, \\ Vívian Oberhofer Ribeiro \\ Coimbra ${ }^{2}$ \\ Vanessa Chaia Kaippert ${ }^{3}$, \\ Eliane Lopes Rosado ${ }^{4}$
}

${ }^{1}$ Ciências Nutricionais. Programa de Obesidade e Cirurgia Bariátrica do Hospital Universitário Clementino Fraga Filho da Universidade Federal do Rio de Janeiro. Rio de Janeiro, RJ

${ }^{2}$ Nutrição Clínica. Instituto de Nutrição Josué de Castro da Universidade Federal do Rio de Janeiro. Rio de Janeiro, RJ.

${ }^{3}$ Ciências Biológicas. Laboratório de Avaliação Nutricional do Instituto de Nutrição Josué de Castro da Universidade Federal do Rio de Janeiro. Rio de Janeiro, RJ.

${ }^{4}$ Ciências e Tecnologia de Alimentos. Departamento de Nutrição e Dietética do Instituto de Nutrição Josué de Castro da Universidade Federal do Rio de Janeiro. Rio de Janeiro, RJ.

$\triangle$ Fernanda Mattos

Rua Visconde de Pirajá, 414, salas 1104 e 1105, Ipanema CEP: 22410-002

Rio de Janeiro - RJ

fernandamattos.nut@gmail.com

\section{RESUMO}

A obesidade é uma doença crônica e tem se tornado uma epidemia em todo o mundo. Sua etiologia é multifatorial e o tratamento complexo, envolvendo dieta, exercícios físicos, fármacos e, em casos mais graves, cirurgia. De acordo com guias e consensos nacionais e internacionais para tratamento nutricional da obesidade, a distribuição dos macronutrientes pode variar de $35 \%$ a $65 \%$ do valor energético total (VET) para carboidratos, de 15 a $25 \%$ do VET para proteínas e de 20 a $40 \%$ do VET para lipídios. Em relação aos lipídios, verificou-se que os aspectos qualitativos são mais relevantes, com a redução na ingestão de colesterol, gorduras saturadas e trans e ênfase nos lipídios insaturados. É recomendando o consumo de carboidratos complexos e fibras dietéticas. Dietas restritivas não se mostram sustentáveis por longos períodos, mas podem ser úteis em situações específicas por um período limitado. Indivíduos obesos apresentam alta prevalência de deficiência de micronutrientes, inclusive antes da cirurgia bariátrica, devido a hábitos alimentares inadequados e outros fatores. 0 planejamento alimentar do indivíduo com obesidade deve ser flexível e individualizado e deve motivar práticas alimentares adequadas e saudáveis. Para maior sucesso terapêutico é fundamental associar a intervenção nutricional com a prática regular de atividades físicas. A adesão dietética e, consequentemente, o balanço energético negativo são fatores decisivos para a promoção da perda e manutenção de peso corporal.

Palavras-chave: obesidade, macronutrientes, micronutrientes

\section{ABSTRACT}

Obesity is a chronic disease and has become an epidemic all over the world. Its etiology is multifactorial and its treatment is complex, involving diet, physical exercises, drugs and, in more serious cases, surgery. According to national and international guidelines and consensus for nutritional treatment of obesity, the distribution of macronutrients can vary from $35 \%$ to $65 \%$ of the total energy value (TEV) for carbohydrates, from 15 to $25 \%$ of the VET for proteins and from 20 to $40 \%$ of TEV for lipids. In relation to lipids, it was verified that the qualitative aspects are more relevant, with the reduction in cholesterol intake, saturated and trans fats and emphasis on unsaturated lipids. Increasing consumption of complex carbohydrates and dietary fibers is recommended. Restrictive diets are not sustainable for long periods, but may be useful in specific situations for a limited period. Obese individuals have a high prevalence of micronutrient deficiency, even before bariatric surgery, due to inadequate eating habits and other factors. Food planning of the individual with obesity should be flexible and individualized and should motivate adequate and healthy eating practices. For greater therapeutic success, it is paramount to associate the nutritional intervention with the regular practice of physical activities. Dietary adherence and, consequently, negative energy balance are decisive factors to ensure weight loss and body weight maintenance.

Keywords: obesity, macronutrients, micronutrients 


\section{INTRODUÇÃO}

A prevalência de obesidade continua aumentando mundialmente sendo um dos problemas de saúde pública mais desafiadores. Dados recentes mostram que a porcentagem de pessoas com obesidade dobrou em mais de 70 países entre 1980 e 2015 (AFSHIN et al., 2017). Em 2016, mais de 1,9 bilhões de indivíduos apresentavam excesso de peso no mundo, dos quais mais de 650 milhões eram obesos (WHO, 2018).

Indivíduos com obesidade estão sob grande risco de desenvolver graves comorbidades, incluindo doenças cardiovasculares (DCV), distúrbios gastrointestinais, diabetes mellitus tipo 2 (DM2), doença do refluxo gastro esofágico (DGRE), esteatose hepática, degeneração e/ ou agravamento de distúrbios articulares e musculares, problemas respiratórios e psicológicos, certos tipos de câncer, entre outras, que afetam significativamente a qualidade de vida e aumentam os riscos de mortalidade (FRUSH, 2017).

Basicamente, existem três abordagens para o tratamento da obesidade: dietética, farmacológica e cirúrgica. Tais estratégias podem ser indicadas de forma combinada ou não, de acordo com a gravidade do problema e com a presença ou ausência de complicações associadas. Porém, mudanças de estilo de vida incluindo hábitos alimentares saudáveis e prática regular de atividade física são fundamentais para obtenção de sucesso terapêutico em curto, médio e longo prazo (ABESO, 2016).

Estudos mostram que a perda ponderal de $5 \%$ a $10 \%$ em indivíduos com excesso de peso ou obesidade com comorbidades, apresenta um impacto positivo sobre a saúde global e melhora metabólica (WADDEN et al., 2014; MAGKOS etal, 2016; FRUSH, 2017).

A prescrição dietética é um dos pilares no tratamento da obesidade independentemente do grau do índice de massa corporal (IMC), devendo estar de acordo com a ingestão dietética diária recomendada (IDR) a fim de garantir o consumo adequado de macro e micronutrientes de acordo com as necessidades de cada indivíduo (JENSENet al.,2014; ABESO, 2016).

Nesse sentido, o presente artigo tem como objetivo contribuir para o tratamento dietético da obesidade, por meio da revisão crítica das recomendações de macro e micronutrientes para obesidade e dos resultados de pesquisas científicas recentes.

\section{REVISÃO DE LITERATURA}

\section{Energia}

Na prática clínica e em grande parte dos estudos científicos são utilizadas equações preditivas para estimar as necessidades energéticas individuais. No entanto, a maioria destas equações preditivas foi desenvolvida para populações saudáveis ou com outros tipos de enfermidades e de outras etnias (europeias e norte-americanas, principalmente). Estudos que avaliam a eficácia destas fórmulas para estimar as necessidades energéticas em obesos são contraditórios, mostrando que estas podem superestimar ou subestimar as calorias a serem prescritas, conforme as equações avaliadas (SCHUDZIARRA et al., 2014; ROSADO et al., 2014; MARRA et al., 2017). Nesse sentido, algumas estratégias práticas individuais foram elaboradas de forma a auxiliar a prescrição energética de pacientes com obesidade.

O Consenso Latinoamericano de Obesidade (COUTINHO et al.,1999) já recomendava a redução progressiva da ingestão calórica de 500 a $1.000 \mathrm{kcal} / \mathrm{dia}$ com relação ao valor energético obtido a partir da anamnese alimentar.

O guia americano para o tratamento da obesidade (JENSEN et al.; 2014) sugere: 1) Prescrição de 1.200 a $1.500 \mathrm{kcal} / \mathrm{dia}$ para as mulheres e 1.500 a $1.800 \mathrm{kcal} /$ dia para os homens. Ressaltando que estas prescrições poderão ser ajustadas de acordo com a massa corporal e nível de atividade física do indivíduo. 2) Déficit calórico de $500 \mathrm{kcal} /$ dia a $750 \mathrm{kcal} / \mathrm{dia}$ ou de $30 \%$, com base nas preferências individuais e estado de saúde do paciente. 3) Abordagem ad libitum sem prescrição formal de déficit de energia, na qual a redução da ingestão calórica é obtida pela restrição ou eliminação de grupos de alimentos específicos ou prescrição de alimentos.

O consenso europeu de obesidade (YUMUK et al., 2015) propõe: 1) Déficit diário de $600 \mathrm{kcal}$ a fim de alcançar perda de $0,5 \mathrm{~kg}$ por semana. 2) Redução de $15-30 \%$ na ingestão de calorias da ingestão habitual. 3) Regra prática para estimativa da necessidade diária de energia considerando $25 \mathrm{kcal} / \mathrm{kg}$ para ambos os sexos. 4) Dietas de baixa caloria (800 a $1.200 \mathrm{kcal}$ ao dia) e dietas de muito baixa caloria (menos que $800 \mathrm{kcal}$ ao dia), sendo as mais restritivas utilizadas somente por curto período de tempo e sob supervisão de um especialista em obesidade.

As diretrizes brasileiras de obesidade (ABESO, 2016) sugerem: 1) Prescrição de 1.000 a $1.500 \mathrm{kcal} /$ dia para as mulheres e 1.200 a $1.800 \mathrm{kcal} /$ dia para os homens. 2) Dieta planejada individualmente para criar um déficit calórico de $500 \mathrm{kcal} /$ dia a $1.000 \mathrm{kcal} / \mathrm{dia}$ a fim de alcançar a perda de $0,5 \mathrm{~kg}$ a $1 \mathrm{~kg}$ por semana. 3) Dietas de muito baixa caloria com oferta energética de 400 a $800 \mathrm{kcal}$ por dia, desde que realizadas em ambiente hospitalar sob rigorosa supervisão médica. Vale destacar que estas dietas podem levar a maior perda de peso em curto prazo quando comparadas às dietas de baixas calorias, porém, em longo prazo a perda de peso é semelhante.

Os guias citados anteriormente corroboram ao sugerirem déficit calórico para perda de peso. No entanto, não exibem de forma clara como deve ser feita essa redução. Porém, todas as fontes consultadas citam de forma contundente a importância de se avaliar a ingestão calórica habitual, sugerindo que tal redução deverá ser aplicada sobre a ingestão energética habitual do indivíduo. 


\section{Macronutrientes}

A distribuição adequada dos macronutrientes (carboidratos, proteínas e lipídios) em relação ao percentual de calorias para indivíduos obesos não está totalmente esclarecida na literatura. A tabela 1 apresenta as recomendações de macronutrientes propostas pelo Consenso Latinoamericado (COUTINHO et al, 1999), pelo guia americano (JENSEN et al.; 2014) e pelas diretrizes brasileiras para obesidade (ABESO, 2016). O consenso europeu de obesidade (YUMUK et al., 2015) não inclui informação a respeito da distribuição dos macronutrientes da dieta.

O consumo de fibras dietéticas deve ser estimulado em pacientes obesos uma vez que estas possuem várias funções importantes para o bom funcionamento

Tabela 1: Distribuição dos macronutrientes apresentados nos guias de obesidade

\begin{tabular}{|c|c|c|c|}
\hline & $\begin{array}{c}\text { Consenso Latinoamericano } \\
\text { (COUTINHO et al.,1999) }\end{array}$ & $\begin{array}{c}\text { Guia Americano } \\
\text { (JENSEN et al.,2014) }\end{array}$ & $\begin{array}{c}\text { Diretriz Brasileira } \\
(\text { ABESO, 2016) }\end{array}$ \\
\hline Carboidratos & $55 \%$ a $60 \%$ & $35 \%$ a $65 \%$ & $55 \%$ a $60 \%$ \\
\hline Proteínas & $\begin{array}{c}15 \% \text { a } 20 \% \\
\text { ou } \\
\text { 0,8g/proteínas/peso } \\
\text { desejável }\end{array}$ & $15 \%$ a $25 \%$ & $15 \%$ a $20 \%$ \\
\hline Lipídios & $20 \%$ a $25 \%$ & $20 \%$ a $40 \%$ & $20 \%$ a $30 \%$ \\
\hline
\end{tabular}

do organismo, tais como: auxiliam na redução da ingestão alimentar por meio do aumento do tempo de mastigação; retardam o esvaziamento gástrico; auxiliam na diminuição da secreção de insulina; aumentam a sensação de saciedade; contribuem para o bom funcionamento e integridade do intestino e, ainda, atuam no controle das concentrações séricas de colesterol e glicose (IOM, 2005). Nesse sentido, as recomendações do consumo de fibras variam de 20 a $30 \mathrm{~g}$ de fibras totais/dia de acordo com o Consenso Latino Americano de Obesidade (1999).

Embora exista forte relação entre os lipídios dietéticos e a obesidade, nenhum dos documentos de referência consultados sugere recomendações para consumo dos diferentes tipos de lipídios dietéticos: saturados, monoinsaturados, poli-insaturados e trans.

Alguns autores preconizam programas alimentares de normo a hiperproteicos para favorecer a perda de peso, aumentar a saciedade por meio da liberação de peptídeo semelhante ao glucagon 1 (GLP1) e polipeptideo yy (PYY) e a termogênese induzida pela dieta VAN DER KLAAUM et al., 2013; TULLOCH et al., 2015). Entretanto, dietas hipocalóricas (restrição de 500 a $750 \mathrm{kcal} / \mathrm{dia}$ ) ricas em proteínas (25\% do valor energético total [VET]) resultam em perda de peso semelhante a dietas normoproteicas (15\% do VET) (JENSEN et al., 2014). Importante ressaltar que dietas com alto teor de proteína devem ser prescritas com cautela, uma vez que sua ingestão pode afetar o balanço dos lipídios, principalmente de lipídios saturados, presentes naturalmente nas fontes de proteína animal.

\section{Micronutrientes}

Estudos mostram que deficiências de vitaminas e minerais são muito comuns em obesos, podendo ser encontradas baixas concentrações de vários micronutrientes (VALENTINO; SRIRAM; SHANKAR, 2011; VIA;MECHANICK, 2017). Modificações nos padrões alimentares que vem ocorrendo nas últimas décadas, incluindo o consumo excessivo de alimentos industrializados e a ingestão insuficiente de frutas, legumes e verduras, contribuem para estas deficiências nutricionais. Convém salientar que diversos micronutrientes estão envolvidos em importantes processos metabólicos e endócrinos e que estes são fundamentais como fatores de proteção para o desenvolvimento da obesidade e outras doenças crônicas não transmissíveis (LEÃO; SANTOS, 2012).

Para adequação do consumo de micronutrientes da dieta de indivíduos com obesidade devem ser consideradas as Dietary Reference Intakes (DRIs) publicadas pelo Food and Nutrition Board do Institute of Medicine entre os anos de 1997 e 2011 (www.nap. edu), considerando-se os valores de ingestão dietética recomendada (Recommended Dietary Allowance, RDA), de acordo com faixa etária e sexo.

A tabela 2 apresenta as deficiências de vitaminas e minerais mais comuns em indivíduos com obesidade, suas prováveis causas e consequências principais. 
Tabela 2: Considerações sobre deficiências de micronutrientes em obesos

\begin{tabular}{|c|c|c|}
\hline Nutrientes & Considerações & Autores \\
\hline Cálcio & $\begin{array}{l}\text { - Alto consumo de bebidas açucaradas e redução da } \\
\text { ingestão de leite. } \\
\text { - Elevação do hormônio da paratireoide. }\end{array}$ & $\begin{array}{l}\text { PERSON et al., } 2008 \\
\text { LIMA et al., } 2013 \\
\text { KAIDAR- ROUST; DIBAISE, } 2017 \\
\text { KRZIZEK et al., } 2017\end{array}$ \\
\hline Vitamina D & $\begin{array}{c}\text { - Baixa exposição solar e deficiência relacionada com } \\
\text { doenças ósseas e estágios avançados de fibrose hepática. } \\
\text { - Retenção de vitaminas lipossolúveis pelo tecido adiposo. } \\
\text { - Relação inversa com IMC elevado. }\end{array}$ & $\begin{array}{l}\text { LIMA et al., } 2013 \\
\text { WOLF et al., } 2015 \\
\text { KRZIZEK et al., } 2017\end{array}$ \\
\hline Tiamina & - Elevado consumo de carboidratos refinados e lipídios. & LIMA et al., 2013 \\
\hline Betacaroteno & $\begin{array}{l}\text { - Consumo insuficiente de frutas, verduras e legumes e } \\
\text { retenção pelo excesso de tecido adiposo. }\end{array}$ & WOLF et al., 2015 \\
\hline Ferro & $\begin{array}{c}\text { - Alteração da homeostase do ferro causada pelo estado } \\
\text { pró inflamatório do tecido adiposo. } \\
\text { - Baixa ingestão de carnes. } \\
\text { - Deficiência leva a alterações nos níveis séricos de } \\
\text { ferritina e hemoglobina. }\end{array}$ & $\begin{array}{l}\text { LIMA et al., } 2013 \\
\text { AIGNER; FELDMAN; DATZ, } 2014 \\
\text { WOLF et al., } 2015\end{array}$ \\
\hline
\end{tabular}

- Baixa ingestão de fontes alimentares.

Vitamina C e Selênio - Estresse oxidativo induzido pela inflamação crônica eleva ～WOLF et al., 2015 o requerimento de antioxidantes.

- Baixa ingestão de vegetais folhosos, legumes e produtos integrais e baixa capacidade de armazenamento corporal.

Ácido fólico _ - Deficiência afeta a síntese e reparo de DNA, processo

de divisão e crescimento celular, formação do glóbulos

WOLF et al., 2015

FRAME-PETERSON et al., 2017

vermelhos, metabolismo de aminoácidos, desenvolvimento

do tubo neural do feto, e pode levar à anemia.

- Baixo consumo de carnes, ovos e produtos lácteos,

Vitamina B12

interações medicamentosas (exemplo: metformina)

- Pode levar a doenças hematológicas e neurológicas

- Retenção de vitaminas lipossolúveis pelo tecido adiposo.

- Baixa ingestão de frutas, verduras e legumes, leite, fígado e ovos.

Vitamina A - Deficiência pode levar a aumento do estresse oxidativo, resistência à insulina, prejuízo no metabolismo da glicose,

KAIDAR-PERSON et al., 2008 WOLF et al., 2015 cegueira noturna, redução do controle de infecções e problemas reprodutivos.

- Deficiência está associada ao aumento do risco para DM2.

WOLF et al., 2015

FRAME-PETERSON et al., 2017

alimentar do paciente. Contudo, o nutricionista deve

\section{DISCUSSÃO}

Um dos primeiros passos para definir o planejamento alimentar de um indivíduo, independente do diagnóstico nutricional, é estimar suas necessidades energéticas por meio de equações preditivas, visto que métodos mais precisos como calorimetria indireta normalmente não estão disponíveis na prática clínica. Em geral, em pacientes com excesso de peso, esse resultado está muito distante de sua ingestão alimentar habitual. Portanto, na prática clínica, é mais comum se aplicar determinado déficit calórico sobre a ingestão alimentar habitual por meio de mudanças qualitativas no consumo sempre considerar a possibilidade de subnotificação do consumo energético, prática comum nos pacientes obesos (YUMUK et al., 2015).

Pesquisadores em todo mundo têm testado variações na distribuição de macronutrientes em busca da dieta ideal para promoção de perda de peso, incluindo: dietas com baixo teor de lipídios, dietas com baixo teor de carboidratos ou ricas em proteínas, dietas de baixo índice glicêmico, dietas hipocalóricas balanceadas, dietas vegetarianas ou veganas e ainda padrões dietéticos que eliminam um ou mais grupos de alimentos. Independente da estratégia adotada, 
resultados mostram que nenhum padrão dietético é capaz de promover maior perda de peso se não promover balanço energético negativo. Portanto, cabe ao nutricionista buscar adequar o planejamento alimentar do paciente às suas preferências alimentares e assim garantir maior adesão. Não significa que a composição da dieta não seja importante, e sim que a adesão dietética, e, consequentemente, o balanço energético negativo são fatores decisivos para a promoção da perda de peso (JENSEN et al., 2014; RYAN; KAHAN, 2018).

Conforme proposto nas diretrizes brasileiras para tratamento da obesidade, o planejamento alimentar deve ser individualizado, levando em consideração o estado de saúde e as preferências do paciente a fim de garantir maior aderência, mantendo-se déficit energético de 500 a $1.000 \mathrm{kcal} /$ dia e a adequação de nutrientes $(20 \%$ a $30 \%$ de lipídios, $55 \%$ a $60 \%$ de carboidratos e $15 \%$ a $20 \%$ de proteínas, e aporte adequado de vitaminas e minerais) (ABESO, 2016).

Quanto à oferta de proteínas, o nutricionista deve estar ciente que a estimativa das necessidades individuais baseada na massa corporal $(0,8 \mathrm{~g}$ de proteínas $/ \mathrm{kg} / \mathrm{dia}$ ) pode levar a um plano alimentar hiperproteico ( $>25 \%$ do VET), embora esta não seja a meta principal. Portanto, a determinação do conteúdo de proteínas em porcentagem do VET pode ser mais apropriada.

Em relação às dietas com alto teor de proteína, estudos demonstram desfechos positivos em obesos, especialmente nos que possuem DM2 (CLIFTON, 2012) e dislipidemias (MAKRIS; FOSTER, 2011). Há dados que indicam maior adesão à dieta, redução da massa gorda, das concentrações de triglicerídeos, da pressão arterial (MAKRIS; FOSTER, 2011), da glicose sérica e melhora da hemoglobina glicada (CLIFTON, 2012). O teor de proteínas se mostrou variável nos estudos: $1,6 \mathrm{~g} / \mathrm{kg} / \mathrm{dia}$ (MAKRIS; FOSTER, 2011) e 1 a 2g/kg/dia (WESTERTERP-PLANTENGA; LEMMENS; WESTERTERP, 2012).

Os resultados apontam que uma dieta normo a hiperproteica pode aumentar a saciedade e auxiliar na promoção da perda de peso. Contudo, não foi esclarecido nesses estudos se a redução do peso corporal e melhora do perfil metabólico estão relacionadas à proteína em si, ao aumento dos lipídios poli-insaturados ou ao maior consumo de fibras (CLIFTON, 2012).

As dietas pobres em carboidrato, por sua vez, não têm uma definição estabelecida pela Food and Drug Administration (FDA). Na maioria dos trabalhos científicos são ofertados de 20 a $50 \mathrm{~g}$ de carboidratos por dia, o equivalente a aproximadamente $10 \%$ das calorias totais diárias (MAKRIS; FOSTER, 2011). Estudos demonstram que tais dietas são de difícil adesão e há poucas evidências da sua segurança em longo prazo (MAKRIS; FOSTER, 2011; ROLLAND et al., 2009). Autores sugerem que são eficazes no controle do peso corporal porque são relativamente ricas em proteína (WESTERTERPPLANTENGA; LEMMENS; WESTERTERP, 2012).

Em relação aos lipídios, alguns autores preconizam de 20 a 35\% do VET, além da redução da ingestão de colesterol, gorduras saturadas e trans. Dietas com menor teor de lipídios ( $20 \%$ do VET) têm se mostrado eficazes na redução e no controle de peso em curto e longo prazo, desde que haja boa adesão (MAKRIS; FOSTER, 2011; RYAN; KAHAN, 2018). Importante ressaltar que dietas com oferta muito reduzida de lipídios podem de lipídios pode ocasionar, em longo prazo, deficiência de ácidos graxos essenciais.

Não é apenas a distribuição de macronutrientes que deve ser considerada durante a elaboração do planejamento alimentar do indivíduo obeso. Tão importante quanto atingir as recomendações totais de carboidratos e lipídios é assegurar sua qualidade, uma vez que dietas com maior proporção de carboidratos complexos e lipídios insaturados podem influenciar a saciedade e o controle metabólico do indivíduo. No entanto, essa questão fundamental não é abordada nos guias para tratamento da obesidade. Semelhantemente, os documentos de referência não destacam os possíveis efeitos benéficos da oferta adequada de fibras dietéticas.

Nos últimos anos, estudos têm buscado avaliar os efeitos da qualidade dos lipídios dietéticos no tratamento da obesidade, porém, os resultados ainda são controversos.

Pesquisas apontam que os ácidos graxos podem influenciar o balanço entre ingestão e gasto energético atuando em diferentes frentes relacionadas à adipogênese, como por exemplo, o gasto energético de repouso, a termogênese induzida pela dieta, a oxidação de substratos e a saciedade, e ainda reduzir a massa corporal e/ou a deposição de gorduras no tecido adiposo (PIERS et al., 2003; CASAS-AGUSTENCH et al., 2009; QUEIROZ et al., 2009; MUNRO; GARG, 2012). Dados mostram que os ácidos graxos insaturados podem favorecer o controle de complicações associadas à obesidade como resistência à insulina, dislipidemias e síntese aumentada de marcadores inflamatórios pelo tecido adiposo (RAMEL et al., 2008; ITARIU et al., 2012; URPI-SARDA et al., 2012).

Em função desses achados, nos últimos anos, as recomendações nutricionais práticas em relação ao consumo de lipídios deixaram de ser restritivas e passaram a ter caráter mais qualitativo. Como não existem recomendações de lipídios específicas para o tratamento da obesidade, podem ser utilizados os valores propostos pela Food and Agriculture Organization of the United Nations (FAO, 2010), pelo Institute of Medicine (IOM, 2005) e outros documentos técnicos mais recentes como a I Diretriz sobre o Consumo de Gorduras e Saúde Cardiovascular (SANTOS et al., 2013). No quadro 1 estão sintetizadas tais recomendações.

A alta prevalência de desnutrição em indivíduos 
Quadro 1: Recomendações dietéticas para lipídios totais e frações

\begin{tabular}{|lccc|}
\hline & FAO (2010) & IOM (2005) & Santos et al. (2013) \\
\hline Lipídios totais & 20 a $35 \%$ VET & 20 a $35 \%$ VET & - \\
AGS & $<10 \%$ VET & - & $<\%$ VET \\
AGPI & 6 a $11 \%$ VET & - & - \\
AGPI $n-3$ & 0,5 a $2 \%$ VET & 0,6 a $1,2 \%$ VET & - \\
AGPI $n-6$ & 2,5 a $9 \%$ VET & 5 a $10 \%$ VET & $10 \%$ VET \\
EPA + DHA & 0,250 a 2 g & - & - \\
AG Trans & $<1 \%$ VET & - & $<1 \%$ VET \\
AGMI & 15 a $20 \%$ VET* & - & - \\
\hline
\end{tabular}

VET: valor energético total; AG: ácidos graxos; AGS: ácidos graxos saturados; AGPI: ácidos graxos poli-insaturados; AGPI n-3: ácidos graxos poli-insaturados ômega-3; AGPI n-6: ácidos graxos poli-insaturados ômega-6; EPA: ácido eicosapentaenoico; DHA: ácido docosahexaenoico; AGMI: ácidos graxos monoinsaturados.

* Lipídios totais (\% VET) - AGS (\% VET) - AGPI (\% VET) - AG Trans (\% VET)

com excesso de peso e obesidade é demonstrada na literatura científica (KAIDAR-PERSON et al., 2008; WOLF et al., 2015). As causas apresentadas são multifatoriais e incluem dieta de baixo valor nutricional (ROUST; DIBAISE, 2017) e aumento da adiposidade corporal, que influencia o armazenamento e a biodisponibilidade de alguns nutrientes, especialmente vitaminas lipossolúveis e antioxidantes (LIMA et al., 2013). Estudiosos destacam que as principais deficiências de micronutrientes verificadas nessa população são cálcio, vitaminas $A$, D (ROUST; DIBAISE, 2017), C, B12 (THOMAS-VALDÉS et al., 2016), tiamina, ferro, betacaroteno (WOLF et al., 2015), ácido fólico (FRAME-PETERSON et al., 2017; KRZIZEK et al., 2017), selênio e zinco (LIMA et al., 2013).

Mais estudos são necessários para avaliar se a deficiência de vitaminas é causa ou consequência do excesso de tecido adiposo (THOMAS-VALDÉS et al., 2016). É consenso que o rastreamento da deficiência de micronutrientes e tratamento precoce por meio de atendimento individualizado são importantes para prevenir futuras complicações (FRAME-PETERSON et al., 2017; ROUST; DIBAISE, 2017; RYAN; KAHAN, 2018). As diretrizes que tratam do manejo dietético na obesidade não são mandatórias e devem ser interpretadas com base no julgamento clínico (RYAN; KAHAN, 2018).

A insuficiência múltipla de vitaminas e minerais descrita acima também acomete candidatos à cirurgia bariátrica, visto que em sua maioria possuem obesidade mórbida (KRZIZEK et al., 2017; LIMA et al., 2013; WOLF et al., 2015). Há concordância científica de que a avaliação do estado pré-operatório é primordial para auxiliar na suplementação antes e após a cirurgia bariátrica e evitar maiores complicações no período pósoperatório (ROUST; DIBAISE, 2017; WOLF et al., 2015).

Em relação à problemática apresentada, a solução não é o consumo excessivo de suplementos vitamínicos e minerais, e sim educação nutricional e mudanças no estilo de vida (KAIDAR-PERSON et al., 2008).

Acima de tudo é dever do nutricionista buscar motivar o indivíduo a realizar práticas alimentares adequadas e saudáveis conforme recomendações do Guia Alimentar para a População Brasileira: dar preferência a alimentos in natura e minimamente processados, priorizando os de origem vegetal; limitar o consumo de alimentos processados; utilizar ingredientes culinários com moderação (óleos, gorduras, sal e açúcar); e evitar o consumo de ultraprocessados. Aspectos relacionados ao comportamento alimentar também devem ser trabalhados junto ao paciente: comer com regularidade e atenção; comer em ambientes apropriados; comer em companhia e compartilhar habilidades culinárias (BRASIL, 2014).

Um dos principais desafios do tratamento da obesidade é assegurar a manutenção da massa corporal em longo prazo. De modo geral, pacientes obesos perdem peso quando mantidos em dieta hipocalórica, entretanto, são incapazes de manter o peso corporal em longo prazo. Cerca de $50 \%$ dos obesos recuperam o peso pré-tratamento em um ano e apenas $11 \%$ são capazes de manter a perda de $5 \mathrm{~kg}$ ou mais (ABESO, 2016).

Programas de intervenção no estilo de vida podem favorecer a perda e manutenção do peso perdido. Os principais componentes de intervenções efetivas incluem: prescrição dietética com redução calórica moderada; aumento da atividade física; e o uso de estratégias comportamentais para facilitar a aderência à dieta e exercícios físicos (JENSEN et al., 2014).

Minimamente deve-se buscar a associação da intervenção nutricional com a prática regular de atividades físicas orientada por um educador físico. O tratamento dietético é mais bem sucedido quando aliado a um programa de modificação comportamental que envolva aumento do gasto energético, promovendo maior balanço energético negativo.

Ademais, é importante lembrar que para o sucesso do tratamento dietético, devem-se manter mudanças na alimentação por toda a vida, portanto, dietas muito restritivas 
não são sustentáveis, embora possam ser usadas por um período limitado de tempo. Um planejamento alimentar mais flexível, que considere as preferências alimentares, o aspecto financeiro e o estilo de vida do paciente, e que objetive acima de tudo a reeducação alimentar e nutricional, geralmente obtém mais sucesso em curto e longo prazo. 0 contato frequente entre o nutricionista e o paciente também pode contribuir fortemente para a perda e a manutenção do peso perdido (ABESO, 2016).

\section{CONCLUSÃO}

O planejamento alimentar do paciente obeso deve ser individualizado, levando em consideração seu estado de saúde e preferências individuais a fim de garantir maior aderência, mantendo-se déficit energético que favoreça a perda de peso e adequação de nutrientes não só do ponto de vista quantitativo, mas, principalmente, prezando pela qualidade da alimentação.

É importante lembrar que para o sucesso do tratamento dietético, devem-se manter mudanças na alimentação por toda a vida, portanto, dietas muito restritivas não são sustentáveis, embora possam ser usadas por um período limitado de tempo. Um planejamento alimentar mais flexível, que considere as preferências alimentares, o aspecto financeiro e o estilo de vida do paciente, e que objetive acima de tudo a reeducação alimentar e nutricional, geralmente obtém mais sucesso em curto e longo prazo. O contato frequente entre o nutricionista e o paciente também pode contribuir fortemente para a perda e a manutenção do peso perdido.

\section{Conflito de interesse}

Os autores declaram não haver conflitos de interesses.

\section{REFERÊNCIAS}

AIGNER, E.; FELDMAN, A.; DATZ, C. Obesity as an emerging risk factor for iron deficiency. Nutrients, v. 6, n. 9, p. 3587-3600, 2014.

AFSHIN, A. et al. Health effects of overweight and obesity in 195 countries over 25 years. New England Journal of Medicine. v.317, n.1, p.1-15, jul. 2017.

ABESO. Associação Brasileira para Estudo da Obesidade e Síndrome Metabólica. Diretrizes brasileiras de obesidade 2016. Disponível em: http://www.abeso.org.br/uploads/ downloads/92/57fccc403e5da.pdf.

BRASIL. Ministério da Saúde. Secretaria de Atenção à Saúde. Departamento de Atenção Básica. Guia alimentar para a população brasileira / Ministério da Saúde, Secretaria de Atenção à Saúde, Departamento de Atenção Básica. - 2. ed., 1. reimpr. Brasília : Ministério da Saúde, 2014. 156 p.: il.
CASAS-AGUSTENCH, P. et al. Acute effects of three high-fat meals with different fat saturations on energy expenditure, substrate oxidation and satiety. Clinical Nutrition, v. 28, p. 39-45, feb. 2009.

CLIFTON, P. Effects of a high protein diet on body weight and comorbidities associated with obesity. British Journal of Nutrition, v. 108, 2012.

COUTINHO, W. et al. Consenso latino-americano de obesidade. Arquivos Brasileiros de Endocrinologia \& Metabologia, v.43, n.1, p.21-67, feb. 1999.

FAO. Food and Agriculture Organization of the United Nations. Fats and fatty acids in human nutrition. Report of an expert consultation. Rome: FAO, 2010. 189 p. Disponível em: https:// www.who.int/nutrition/publications/nutrientrequirements/ fatsandfattyacids_humannutrition/en/.

FRAME-PETERSON, L. A. et al. Nutrient deficiencies are common prior to bariatric surgery. Nutrition in Clinical Practice, v. 32, n. 4, p. 463-469, 2017.

FRUH, S.M. Obesity: risk factors, complications, and strategies for sustainable long-term weight management. Journal of the American Association of Nurse Practitioners. v.29, n.1, p.3-14, oct. 2017.

FUKUNAKA, A.; FUJITANI, Y. Role of zinc homeostasis in the pathogenesis of diabetes and obesity. International Journal of Molecular Sciences, v. 19, n. 2, 2018.

GARDNER, C. D. et al. Weight loss on low-fat vs. lowcarbohydrate diets by insulin resistance status among overweight adults and adults with obesity: a randomized pilot trial. Obesity, v. 24, n. 1, p. 79-86, 2016.

ITARIU, B. K. et al. Long-chain n-3 PUFAs reduce adipose tissue and systemic inflammation in severely obese nondiabetic patients: a randomized controlled trial. American Journal of Clinical Nutrition, v. 96, n. 5, p. 1137-1149, nov. 2012.

IOM. Institute of Medicine. Dietary reference intakes for energy, carbohydrate, fiber, fatty acids, cholesterol, protein, and amino acids (macronutrients). The National Whashington, D. C.: Academies Press, 2005. Disponível em: https://www.nal.usda.gov/sites/default/files/fnic_uploads/ energy_full_report.pdf

JENSEN, M.D. et al. 2013 AHA/ACC/TOS guideline for the management of overweight and obesity in adults: a report of the American College of Cardiology/American Heart Association Task Force on Practice Guidelines and The Obesity Society. Journal of the American College of Cardiology. v. 63, n. 25, p. 2985-3023, jul. 2014.

KAIDAR-PERSON, O. et al. Nutritional deficiencies in morbidly obese patients: a new form of malnutrition? Obesity Surgery, v. 18, n. 8, p. $1028-1034,2008$. 
KRZIZEK, E. C. et al. Prevalence of micronutrient deficiency in patients with morbid obesity before bariatric surgery. Obesity Surgery, v. 28, n. 3, p. 643-648, 2017.

LEÃO, A.L.M.; SANTOS, L.C. Consumo de micronutrientes e excesso de peso: existe relação? Revista Brasileira de Epidemiologia, v.15, n.1, p.85-95, mar. 2012.

LIMA, K.V.G. et al. Micronutrient deficiencies in the pre-bariatric surgery. Arquivos Brasileiros de Cirurgia Digestiva, v.26, n.1, p.63-66, dec. 2013.

LU, M. et al. Effects of low-fat compared with high-fat diet on cardiometabolic indicators in people with overweight and obesity without overt metabolic disturbance: a systematic review and meta-analysis of randomised controlled trials. British Journal of Nutrition, v. 119, 2018.

MAGKOS, F. et al. Effects of moderate and subsequent progressive weight loss on metabolic function and adipose tissue biology in humans with obesity. Cell Metabolism, v.23, n.4, p.591-601, apr. 2016.

MAKRIS, A.; FOSTER, G. D. Dietary approaches to the treatment of obesity. Psychiatric Clinics of North America, v. 34, n. 4, p. 813-827, 2011.

MARRA, M. et al. Prediction and evaluation of resting energy expenditure in a large group of obese outpatients. International Journal of Obesity (London), v.41, n.5, p.697-705, May.2017.

MUNRO, I. A.; GARG, M. L. Dietary supplementation with n-3 PUFA does not promote weight loss when combined with a very-lowenergy diet. British Journal of Nutrition, v. 108, p. 1466-1474, oct. 2012.

PIERS, L. S. et al. Substitution of saturated with monounsaturated fat in a 4-week diet affects body weight and composition of overweight and obese men. British Journal of Nutrition, v. 90, n. 3, p. 717-727, sep. 2003.

QUEIROZ, J.C.F. et al. Controle da adipogênese por ácidos graxos. Arquivos Brasileiros de Endocrinologia \& Metabologia, v.53, n.5, p.582-594, jul. 2009.

RAMEL, A. et al. Beneficial effects of long-chain n-3 fatty acids included in an energy-restricted diet on insulin resistance in overweight and obese European young adults. Diabetology, v. 51, n. 7, p. 1261-1268, jul. 2008.

ROLLAND, C. et al. Randomized clinical trial of standard dietary treatment versus a low-carbohydrate/high-protein diet or the Lighter Life Programme in the management of obesity. Journal of Diabetes, v. 1, p. 207-217, 2009.

ROSADO, E.L. et al.Effectiveness of prediction equations in estimating energy expenditure sample of brazilian and spanish women with excess body weight. Nutricion Hospitalaria, v.29, n.3, p. 513-518, mar. 2014.
ROUST, L. R.; DIBAISE, J. K. Nutrient deficiencies prior to bariatric surgery. Current Opinion in Clinical Nutrition and Metabolic Care, v. 20, n. 2, 2017.

RYAN, D. H.; KAHAN, S. Guideline recommendations for obesity management. Medical Clinics of North America, v. 102, n. 1, p. 49-63, 2018.

SANTOS, R. D. et al. Sociedade Brasileira de Cardiologia. I Diretriz sobre o consumo de gorduras e saúde cardiovascular. Arquivos Brasileiros de Cardiologia, v. 100, n. 1, supl. 3, p. 1-40, jan. 2013.

SCHUSDZIARRA, V. et al. Accuracy of resting energy expenditure calculations in unselected overweight and obese patients. Annals of Nutrition and Metabolism, v.65, n. 4, p. 299-309, out. 2014.

VALENTINO, D.; SRIRAM, K.; SHANKAR, P. Update on micronutrients in bariatric surgery. Current Opinion in Clinical Nutrition and Metabolic Care. v.14, n.6, p.635-641, nov.2011.

VIA, M.A.; MECHANICK, J.I. Nutritional and Micronutrient Care of Bariatric Surgery Patients: Current Evidence Update. Current Obesity Reports. v.6, n.3, p.286-296, set. 2017.

THOMAS-VALDÉS, S. et al. Association between vitamin deficiency and metabolic disorders related to obesity. Critical Reviews in Food Science and Nutrition, v. 57, n. 15, p. 3332-3343, 2016.

TULLOCH, AJ. et al. Neural responses to macronutrients: hedonic and homeostatic mechanisms. Gastroenterology, v.48, n.6, p.1205-1218, may. 2015.

URPI-SARDA, M. et al. Virgin olive oil and nuts as key foods of the mediterranean diet effects on inflammatory biomakers related to atherosclerosis. Pharmacological Research, v. 65, n. 6, p. 577583, jun. 2012.

VAN DER KLAAUW, A.A. et al. High protein intake stimulates postprandial GLP1 and PYY release. Obesity (Silver Spring), v.21, n.8, p.1602-1607, aug. 2013.

WADDEN, T.A. et al. Look AHEAD research group. Eight-year weight losses with an intensive lifestyle intervention: the look AHEAD study. Obesity (Silver Spring), v.22, n.1, p.5-13, jan. 2014.

WESTERTERP-PLANTENGA, M. S.; LEMMENS, S. G.; WESTERTERP, K. R. Dietary protein - its role in satiety, energetics, weight loss and health. British Journal of Nutrition, v. 108, 2012.

WHO. World Health Organization. Obesity and overweight. Feb. 2018. Disponível em: http://www.who.int/mediacentre/factsheets/ fs311/en/.

WITJAKSONO, F. et al. Comparison of low calorie high protein and low calorie standard protein diet on waist circumference of adults with visceral obesity and weight cycling. BMC Research Notes, v. 11, n. 674, 2018. 
WOLF, E. et al. Preoperative micronutrient status in morbidly obese patients before undergoing bariatric surgery: results of a cross-sectional study. Surgery for Obesity and Related

Diseases, v. 11, n. 5, p. 1157-1163, sep./oct. 2015.

YUMUK, V. et al. Obesity management task force of the European association for the study of obesity. European guidelines for obesity management in adults. Obesity Facts, v.8, n.6, p.402-424, dec. 2015. 